\title{
Performance Evaluation of Finite-Resolution IR-UWB Signal Detection
}

\author{
Ma Yan \\ The Research Institute of \\ NBC Defense \\ Beijing, P. R. China \\ cole@mail.ustc.edu.cn
}

\author{
Sun Fei \\ EEIS, University of \\ Science and Technology of \\ China \\ Hefei, P. R. China
}

\author{
Li Peng \\ The Research Institute of \\ NBC Defense \\ Beijing, P. R. China
}

\author{
Yin Huarui \\ EEIS, University of \\ Science and Technology of \\ China \\ Hefei, P. R. China
}

\begin{abstract}
Finite-resolution digital receiver is considered to be a potential solution to meet the severe demand of ADC that results from wideband of IR-UWB signal. However, the quantized distortion is totally different from full-resolution quantized signal. In this paper, the finite-resolution quantized signal model is derived. Based on the correlation receiver, estimation and detection performance of finite-resolution and full-resolution digital receiver are analyzed in the paper. The analytical results agree with the numerical results exactly and the template estimation performance using midtread quantizer is better than that using the adjacent midrise quantizer.
\end{abstract}

\section{Keywords-UWB; finite resolution; detection}

\section{INTRODUCTION}

IR-UWB systems utilize low duty cycle pulses to transmit information. For the extremely wide bandwidth of the UWB waveform, the systems result in many attractive properties, e.g., high transmission rate and accurate ranging resolution [1]. However, there are still tremendous challenges of the UWB technology in reaching its potential. The Nyquist sampling for UWB signal is often needed, which demands giga $\mathrm{Hz}(\mathrm{GHz})$ ADC and hence makes implement of the receiver unrealistically because of the unendurable power for high resolution ADC [2]. The tradeoff between performance and complexity has been made and finite-resolution digital UWB receiver with only one- or two-bit ADC were proposed [3][4]. The optimal finite-resolution UWB digital receiver is shown in [4]. A monobit receiver for UWB communication based on matched filter is proposed in [3] and the optimal monobit receiver is given in [5]. A common conclusion from these works is that contrast with finite-resolution receiver, fullresolution receiver is not necessary because the benefit it performs is not worth the extra power consumption and complexity it takes. These works pay much attention to transplant and modify the detection method of full-resolution receivers to finite-resolution receivers but the quantization noise has not been analyzed systemically yet.

Furthermore, the finite-resolution digital receiver is correlation in most cases. Hence the key part of the receiver is the template for signal detection in communication and ranging in localization applications. In [3], the receiver correlates received signal with ideal noiseless received waveform as template. In [6], the sinusoidal wave is employed as template because it is similar with the Gaussian pulse. However, this method does not consider the distortions caused by multipath and antennas. Therefore, full resolution ideal received waveform is difficult to obtain. Moreover, a pre-assumed template without channel information results in suboptimal detection performance.

In this paper, we investigate the performance evaluation of quantization noise generated by uniform ADC and signal detection based on finite-resolution receiver. The distribution of quantization noise is also investigated and verified by simulations. A typical mean matching technique is employed to obtain an appropriate template with great flexibility in dealing with multipath channels. Performances of template estimation and signal detection are derived in closed forms. The analytical results agree with the numerical results exactly and the template estimation performance using midtread quantizer is better than that using the adjacent midrise quantizer because the odd quantization levels can suppress the quantization noise.

\section{SYSTEM MODEL}

The finite-resolution digital receiver is depicted in Fig. 1. After an ideal low pass filter, the received signal is sampled and quantized via a finite-resolution ADC. The template is estimated using the quantized signal and correlates with data signal to detect the transmitted information.

Considering the IR-UWB scenario with one user, denote $h(t)$ as the channel response function. Let $p(t)$ denote a single transmitted signal and $p_{l p f}(t)$ denote the impulse response of LPF with bandwidth $B$ and gain 1 . The received template is

$$
\omega(t)=p(t) * h(t) * p_{l p f}(t)
$$

where * denotes convolution. Therefore, the filtered IRUWB signal can be written as

$$
r(t)=s(t)+n(t)=\sum_{k=0}^{\infty} d_{k} \omega\left(t-k T_{s}\right)+n(t)
$$

where $d_{k} \in\{+1,-1\}$ is the $k$ th transmitted symbol, $T_{s}$ is the pulse period, $n(t)$ is the additive white Gaussian noise (AWGN) with zero-mean and double-sided power spectral density $N_{0} / 2$.

After the $M$-level uniform finite-resolution quantizer with sampling period $T$, the quantized signal can be represented as

$$
a_{k, i}=Q_{M}\left(r_{k, i}\right)=r_{k, i}+e_{k, i}=d_{k} \omega_{i}+n_{k, i}+e_{k, i}
$$




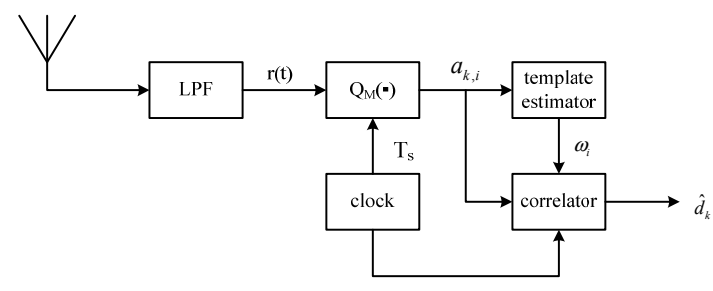

Figure 1. Receiver structure.

where $Q_{M}(\bullet)$ is the quantized function and $i=1, \ldots, N$. Denote $r_{k, i}$ as the $i$ th sample of the $k$ th symbol. $a_{k, i}$ represents the quantized signal and $e_{k, i}$ represents the quantization noise. Every symbol is sampled by $N=T_{s} / T$ points. Note that $\omega_{i}$ is independent of $k$ because of the quasi-static channel. $n_{k, i}$ is the independent and identically distributed (i.i.d.) Gaussian random variable with zero mean and variance $\sigma_{n}^{2}=N_{0} B$.

For the sake of complexity, we obtain the template via $N_{t}$ received symbols as training sequence of pulses, then a correlation digital receiver is used for detecting the unknown information. The template is estimated as

$$
\hat{\omega}_{i}=\frac{1}{N_{t}} \sum_{k=1}^{N_{t}} a_{k, i}
$$

Note that the channel information is contained in the template although it is the dirty template (TDT) [7]. This estimation approach averages signals over multiple symbols, which decreases the estimation error depending on the number of training sequence.

With the template, the decision statistic could be obtained by $\lambda_{k}=\sum_{i=1}^{N} a_{k, i} \hat{\omega}_{i}$ and the transmitted signal is demodulated as $\hat{d}_{k}=\operatorname{sgn}\left(\lambda_{k}\right)$.

\section{PERFORMANCE ANALYSIS}

\section{A. Quantization Noise Statistics}

The quantization noise is distributed as a uniform random variable and independent of the input signal when ADC resolution is high. However, this conclusion is invalid when $\mathrm{ADC}$ resolution is low. The quantization noise is correlated with the input signal.

Consider two types of ADCs: midrise quantizer and midtread quantizer. There are even levels in the midrise quantizer and odd levels in the midtread quantizer. [8]:

The probability density of quantization noise is shown in

$$
f(e)= \begin{cases}\frac{1}{\Delta}\left[1+\sum_{n \neq 0} \alpha_{n} \phi\left(\frac{2 \pi n}{\Delta}\right) \exp \left(\frac{j 2 \pi n e}{\Delta}\right)\right] & -\frac{\Delta}{2} \leq e \leq \frac{\Delta}{2} \\ 0 & \text { otherwise }\end{cases}
$$

where $\Delta=2 V_{m} / M$ is the quantization step and $V_{m}$ is the scale of ADC. Denote $\phi(\varepsilon)=\int_{-\infty}^{+\infty} f(e) \exp (j e \varepsilon) d e$ as the characteristic function of the quantization noise. $\alpha_{n}$ is concerned with $M$.

$$
\alpha_{n}=\left\{\begin{array}{cc}
1 & \text { midtread } \\
(-1)^{n} & \text { midrise }
\end{array}\right.
$$

As mentioned before, $r_{i}=d \omega_{i}+n_{i}$ (due to $r_{k, i}$ is independent, the subscript $k$ is omitted in this subsection). $r_{i}$ is conditionally Gaussian distributed on $d \omega_{i} . n_{i}$ is i.i.d. and $n_{i} \sim N\left(0, \sigma_{n}^{2}\right)$. Define $\mu_{i}^{+}$and $\mu_{i}^{-}$are the expectations corresponding to $d=+1$ and $d=-1$. We obtain

$$
\begin{aligned}
\mu_{i}^{+}= & \frac{\sigma_{n}}{\sqrt{2 \pi}}\left\{\exp \left[-\frac{\left(-V_{m}-\omega_{i}\right)^{2}}{2 \sigma_{n}^{2}}\right]-\exp \left[-\frac{\left(V_{m}-\omega_{i}\right)^{2}}{2 \sigma_{n}^{2}}\right]\right\} \\
& +\omega_{i}\left[Q\left(\frac{-V_{m}-\omega_{i}}{\sigma_{n}}\right)-Q\left(\frac{V_{m}-\omega_{i}}{\sigma_{n}}\right)\right] \\
\mu_{i}^{-} & =\frac{\sigma_{n}}{\sqrt{2 \pi}}\left\{\exp \left[-\frac{\left(-V_{m}+\omega_{i}\right)^{2}}{2 \sigma_{n}^{2}}\right]-\exp \left[-\frac{\left(V_{m}+\omega_{i}\right)^{2}}{2 \sigma_{n}^{2}}\right]\right\} \\
& -\omega_{i}\left[Q\left(\frac{-V_{m}+\omega_{i}}{\sigma_{n}}\right)-Q\left(\frac{V_{m}+\omega_{i}}{\sigma_{n}}\right)\right]
\end{aligned}
$$

Thus, the PDF of quantization noise is (9) on the next page. The mean $E\left(e_{i}\right)$ and second order moment $E\left(e_{i}^{2}\right)$ (or equivalently quantization noise power) of the quantization error can be obtained. Furthermore, the cross-correlation $E\left(r_{i} e_{i}\right)$ between quantization noise and input signal can also be derived. These terms will be employed later.

But the result that the mean and noise power rise as the quantization step goes up (or equally the ADC resolution decreases) can still be explored. Besides, the quantization noise power $E\left(e_{i}^{2}\right)$ increases when the quantization noise variance $\sigma_{n}^{2}$ decreases. In other words, as the SNR goes higher, the impact caused by quantization noise becomes more serious. In addition, when $M$ and $\sigma_{n}^{2}$ are small, quantization noise $e_{i}$ is extraordinarily dependent on the input signal $r_{i}$.

\section{B. Template Estimation Performance}

The estimator generates template using $N_{t}$ training sequence symbols. The template can be written as $\hat{\omega}_{i}=\omega_{i}+\sum_{i=1}^{N_{t}}\left(e_{k, i}+n_{k, i}\right) / N_{t}$. Then the estimation error is $\delta \omega_{i}=\sum_{i=1}^{N_{t}}\left(e_{k, i}+n_{k, i}\right) / N_{t}$.

The mean-square-error(MSE) $\sum_{i=1}^{N} E\left\{\delta \omega_{i}^{2}\right\}$ is used for indicating the estimator performance. 


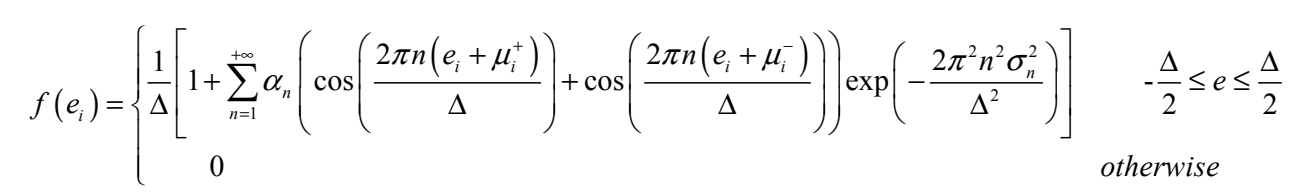

$$
\begin{aligned}
E\left\{\delta \omega_{i}^{2}\right\} & =\frac{1}{N_{t}^{2}} E\left\{\left(\sum_{k=1}^{N_{t}} e_{k, i}\right)^{2}\right\}+\frac{1}{N_{t}^{2}} E\left\{\left(\sum_{k=1}^{N_{t}} n_{k, i}\right)^{2}\right\} \\
& +\frac{2}{N_{t}^{2}} E\left\{\left(\sum_{k=1}^{N_{t}} e_{k, i}\right)\left(\sum_{k=1}^{N_{t}} n_{k, i}\right)\right\}
\end{aligned}
$$

Noting that noise $n_{k, i}$ and quantization noise $e_{k, i}$ are independent. Since both of them are i.i.d., $E\left\{\delta \omega_{i}^{2}\right\}$ can be rewritten as

$$
\begin{aligned}
E\left\{\delta \omega_{i}^{2}\right\} & =\frac{1}{N_{t}}\left\{\sigma_{n}^{2}+2 E\left(r_{i} e_{i}\right)-2 \omega_{i} E\left(e_{i}\right)\right. \\
& \left.+E\left(e_{i}^{2}\right)-\left[E\left(e_{i}\right)\right]^{2}\right\}+\left[E\left(e_{i}\right)\right]^{2}
\end{aligned}
$$

The estimation performance is affected by noise, training sequence length and quantization noise which depends on input signal. Different from the full-resolution template estimator, $E\left\{\delta \omega^{2}\right\}$ can not be reduced to zero by increasing $N_{t}$ or decreasing $\sigma_{n}^{2}$ because of the last term caused by finite-resolution quantization noise.

\section{Signal Detection Performance}

In this subsection, the detection performance is analyzed. We assume that the template is generated by receiving an all "1" training sequence of pulses with the subscript $k$. Then the data information pulses with the subscript $j$ are demodulated and detected by correlating with the estimated template.

The decision statistic can be investigated as

$$
\begin{aligned}
\lambda_{j}= & \sum_{i=1}^{N} a_{j, i} \hat{\omega}_{i} \\
= & \sum_{i=1}^{N}\left[d_{j} \omega_{i}^{2}+\omega_{i} n_{j, i}+\frac{1}{N_{t}}\left(\sum_{k=1}^{N_{t}} n_{k, i}\right) n_{j, i}\right. \\
& +\omega_{i} e_{j, i}+\frac{1}{N_{t}}\left(\left(\sum_{k=1}^{N_{t}} n_{k, i}\right) e_{j, i}+\left(\sum_{k=1}^{N_{t}} e_{k, i}\right) e_{j, i}\right. \\
& \left.\left.+\left(\sum_{k=1}^{N_{t}} e_{k, i}\right) n_{j, i}+d_{j}\left(\sum_{k=1}^{N_{t}} e_{k, i}\right) \omega_{i}+d_{j}\left(\sum_{k=1}^{N_{t}} n_{k, i}\right) \omega_{i}\right)\right] \\
= & d_{j} E_{p}+\xi
\end{aligned}
$$

where $E_{p}=\sum_{i=1}^{N} \omega_{i}^{2}$ and $E_{p}$ is template energy. $\xi$ contains all noise terms including noise, quantization error and so on. $\xi$ is considered as a Gaussian random variable by the central limit theory. Hence we have

$$
m_{\xi}=\sum_{i=1}^{N} \omega_{i} E\left(e_{i}\right)+d_{j} \sum_{i=1}^{N} \omega_{i} E\left(e_{i}\right)+\sum_{i=1}^{N} E\left(e_{i}^{2}\right)
$$

Then the variance is $\sigma_{\xi}^{2}=E\left(\xi^{2}\right)-\{E(\xi)\}^{2}$. The bit error rate is shown as follow

$$
B E R=Q\left[\left(E_{p}+m_{\xi}\right) / \sigma_{\xi}\right]
$$

where $Q_{M}(\cdot)$ is the Gaussian $Q$ function. In order to deeply understand the detection performance gap among different styles of receivers, some special cases are discussed as follow.

1) Full resolution $A D C$ : There is no quantization noise when full-resolution ADC is employed. In this case, we have

$$
m_{\xi}=0, \sigma_{\xi}^{2}=\left(N_{t}+1\right) \sigma_{n}^{2} E_{p}+N \sigma_{n}^{4} / N_{t}
$$

The detection performance of full-resolution receiver is

$$
B E R=Q\left(E_{p} / \sqrt{\left(N_{t}+1\right) \sigma_{n}^{2} E_{p}+N \sigma_{n}^{4} / N_{t}}\right)
$$

This is the performance bound for digital receiver based on finite-resolution estimator.

2) Full-resolution and ideal template: In this case, no quantization noise exists and the template is $\hat{\omega}_{i}=\omega_{i}$. The detection performance is given as

$$
B E R=Q\left(\sqrt{E_{p}} / \sigma_{n}\right)
$$

which is the best detection performance only dominated by background noise. In addition, if $N_{t}$ is very large, (16) becomes (17). In other words, increasing $N_{t}$ can achieve the best detection performance based on the full-resolution receiver.

3) Finite-resolution and ideal template: In this case, the quantization noise exists and the template is known. The decision statistic is $\lambda_{j}=\sum_{i=1}^{N}\left(\omega_{i}^{2}+\omega_{i} n_{j, i}+\omega_{i} e_{j, i}\right)$. We have

$$
m_{\xi}=\sum_{i=1}^{N} \omega_{i} E\left(e_{i}\right)
$$




$$
\begin{aligned}
\sigma_{\xi}^{2} & =\sigma_{n}^{2} E_{p}+\sum_{i=1}^{N} \omega_{i}^{2}\left[E\left(e_{i}^{2}\right)-\left(E\left(e_{i}\right)\right)^{2}\right] \\
& +\sum_{i_{1} \neq i_{2}} \omega_{i_{1}} \omega_{i_{2}}\left[E\left(e_{i_{1}} e_{i_{2}}\right)-E\left(e_{i_{1}}\right) E\left(e_{i_{2}}\right)\right]
\end{aligned}
$$

The detection performance can be expressed as (20) on the next page.

Compared with (16), (14) cannot draw near (20) by increasing $N_{t}$. Due to the impact of quantization noise, the template estimator is not an unbiased estimator. The performance gap between real and ideal template is based on the finite-resolution receiver.

\section{Simulation Results}

In this section, simulations and comparisons are presented. The monopulse $p(t)$ is shaped as a second derivative of a Gaussian pulse with $\tau=0.7 n s$ where $\tau$ is time constant that controls the pulse duration. We set $T_{s}=100 \mathrm{~ns}$ so as to avoid the inter-symbol interference (ISI). Sample period is $T=0.125 n$. The IEEE 802.15.4a channel model 1 (CM1) is employed here.
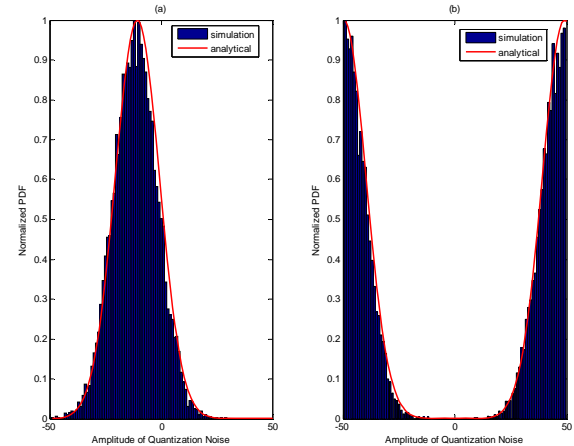

Figure 2. Analytical and numerical PDF of quantization noise generated by a 4-level resolution ADC. Left figure represents the results when amplitude of the input signal is large. Right figure represents the results when amplitude of the input signal is small.
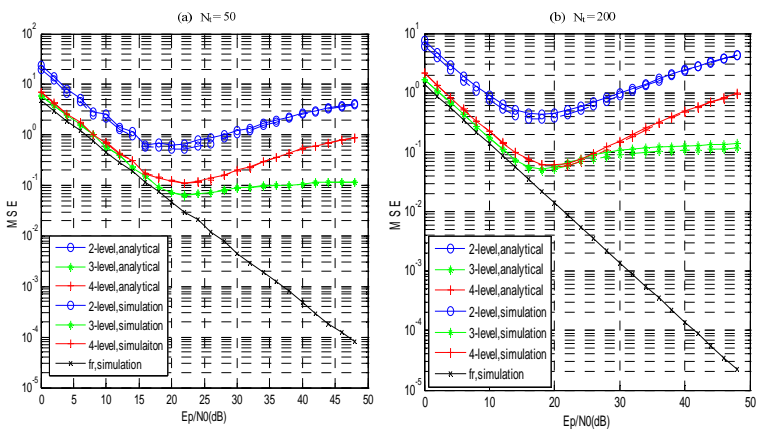

Figure 3. Analytical and numerical MSE versus $N_{t}$, SNR and ADC resolution. Left figure represents MSE when $N_{t}$ is 50 . Right figure represents MSE when $N_{t}$ is 200 .

Fig.2 shows the analytical and numerical PDF curves of quantization noise using a 4-level ADC with different input signal. The analytical results agree with the numerical results exactly. Compared with the two figures, we find that the results are completely different because of the different amplitudes of the input signal. The PDF curves are not symmetric and rely on the input signal when using a finiteresolution ADC.

Fig.3 demonstrates the analytical and numerical MSE curves. The differences among different resolution ADCs become larger when SNR is higher than $25 \mathrm{~dB}$. The curves of the finite-resolution estimator first decline then rise up, while the curves of full-resolution estimator decrease monotonically as the SNR goes up. It is because that channel noise descends and quantization noise grows as SNR rises. We can see that channel noise plays a main role when SNR is low and quantization noise dominates MSE at higher SNR. We can also observe that the higher the resolution ADC is, the lower the convergence level is. Compared with Fig.3a, Fig. $3 \mathrm{~b}$ shows the performances of all types of receivers are improved as $N_{t}$ increases. The curves in different conditions of $N_{t}$ demonstrate that the template estimation performance using midtread quantizer is better than that use the adjacent midriser quantizer because the odd quantization levels can suppress the quantization noise by forcing the small amplitude sampling points to zero.

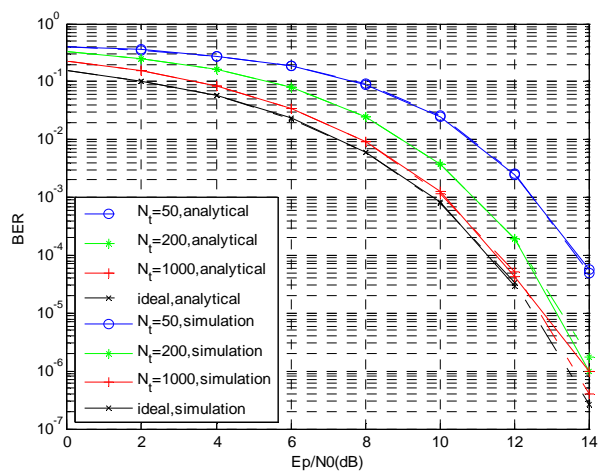

Figure 4. Analytical and numerical BER of full-resolution receiver versus $N_{t}$ and SNR.

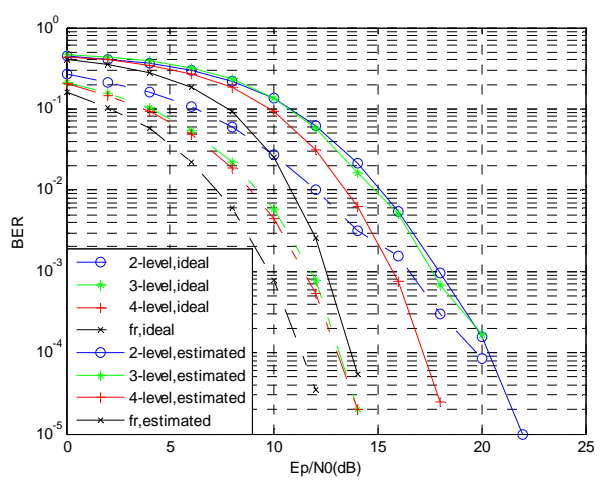

Figure 5. Numerical BER of finite-resolution receiver versus SNR, template and $\mathrm{ADC}$ resolution. 


$$
B E R=Q\left(\frac{E_{p}+\sum_{i=1}^{N} \omega_{i} E\left(e_{i}\right)}{\sqrt{\sigma_{n}^{2} E_{p}+\sum_{i=1}^{N} \omega_{i}^{2}\left[E\left(e_{i}^{2}\right)-\left(E\left(e_{i}\right)\right)^{2}\right]+\sum_{i_{1} \neq i_{2}} \omega_{i_{1}} \omega_{i_{2}}\left[E\left(e_{i_{1}} e_{i_{2}}\right)-E\left(e_{i_{1}}\right) E\left(e_{i_{2}}\right)\right]}}\right)
$$

Fig.4 depicts the analytical and numerical detection performance of full-resolution receiver with different length of training sequence respectively. We observe that the detection performance of full-resolution get close to the best performance based on ideal template by increasing $N_{t}$.

Fig.5 shows the detection results of finite-resolution receiver. We can see that the detection performance is better in higher resolution condition. In addition, it is worth noting that the gap between curves of the same quantizer using ideal and estimated template is very large. That is to say, the detection performances of finite quantized receiver are highly deteriorated by quantization noise contrast with fullresolution receiver.

\section{CONCLUSION}

This paper has investigated detection performance of finite-resolution receiver for IR-UWB which based on the correlation structure. The finite-resolution quantized signal model and distribution of quantization noise are derived. Corresponding estimation and detection performance of the finite-resolution and full-resolution digital receiver are analyzed in closed form. The analytical results agree with the numerical results exactly and the template estimation performance using midtread quantizer is better than that using the adjacent midrise quantizer because the odd quantization levels can suppress the quantization noise.

\section{ACKNOWLEDGMENT}

This work is supported in part by National Science Foundation of China, No.61102011

\section{REFERENCES}

[1] M. Chiani and A. Giorgetti, "Coexistence between UWB and narrowband wireless communication systems," Proc. IEEE, vol. 97, no. 2, pp. 231-254, Feb. 2009

[2] V. Somayazulu, J. Foerster, and S. Roy, "Design challenges for very high data rate UWB systems," Proc. 36th Asilomar Conference on Signals, Systems and Computers, Nov. 2002, vol. 1, pp. 717-721.

[3] S. Hoyos, B. M. Sadler, and G. R. Arce, "Monobit digital receivers for ultrawideband communications," IEEE Trans. on Wireless Commun., vol. 4, no. 4, pp. 1337-1344, Apr. 2005.

[4] L. Ke, H. Yin, W. Gong, and Z. Wang, "Finite-resolution digital receiver design for impulse radio ultra-wideband communication," IEEE Trans. Wireless Commun., vol. 7, no. 12, pp.5108-5117, 2008.

[5] H. Yin, Z. Wang, L. Ke, and J. Wang, "Monobit digital receivers: design, performance, and application to impulse radio," IEEE Trans. on Commun., vol.58, no.6, pp.1695-1704, June 2010.

[6] L. Ke, H. Yin, and P. Xu, "A novel channel estimation method for ultra-wideband communications based on OSCR receiver," Proc. IEEE Communications, Circuits and Systems, May 2005, pp. 432 436 Vol. 1,27-30.
[7] L. Yang and G. B. Giannakis, "Timiming UWB signals using dirty templates," IEEE Trans. on Commun., vol. 53, no. 11, pp. 1952-1963, Nov. 2005.

[8] A. B. Sripad and D. L. Snyder, "A necessary and sufficient condition for quantization errors to be uniform and white," IEEE Trans. Acoust., Speech, Signal Processing, vol. 25, no. 5, pp. 442-448, Oct. 1977. 